production are taking place. A new instrument, based on a pneumatic principle, has been developed for measuring the degree of 'raise' on raised fabrics. This instrument shows considerable promise, not only as a means of assessment, but also as a control instrument for raising machinery. The simple method of measuring warp tension in looms has been improved and instruments are undergoing tests in a number of member firms. The method may also have application in the control of tension in sizing, and suitably modified instruments are about to be evaluated in sizing machinery both at the Association and in the premises of member firms.

As mentioned earlier in this article, the work on machinery development has been restricted by internal reorganization. However, another method of controlling vibration in speed-frames has been developed and is in process of being evaluated. As for fluid beds, a full-scale single-pass bed has been constructed and this is being used for measurements of heat transfer and to assess how far laboratory data on resin-baking and dyeing are applicable under conditions approaching those in a practical machine. Apart from this, most of the development work is concentrated on designing a fluid bed suitable for dyeing fabrics constructed of hydrophobic fibres.

The number of staff is almost the same as last year. The total is 261 made up as follows: research staff, 85 ; laboratory and technical assistants, 75 ; engineering, drawing office, and maintenance staff, 60 ; library and administrative staff, 32 ; and canteen staff, 9 .

L. A. Wiseman

' Nature, 179, 1229 (1957).

2 "Rheology of Elastomers", 79 (Pergamon, 1958). Rheologica Acta, No. 2 (in the press).

'J. Text. Inst., 15, 559 (1924).

\title{
DESIGN AND MANAGEMENT OF A NUCLEAR SCIENCE LABORATORY
}

\author{
By Prof. A. J. WOOdALL, O.B.E., C. G. WILSON, Dr. A. L. JONES and D. K. THOMAS \\ Royal Military College of Science, Shrivenham
}

$\mathrm{T}$ HE following description of a laboratory specially designed for teaching nuclear physics and chemistry at the Royal Military College of Science, Shrivenham, may be helpful to educational institu. tions embarking on work in this field. This laboratory has been in full use for approximately two years and it is now possible to make some assessment of the merits of the various special features incorporated in the design.

The laboratory caters for students, who range from young officers reading for science and engineering degrees to more senior officers on specialist courses. They are mostly pressed for time and more concerned with modern applications than with the history of the subject. Accordingly, modern techniques such as electronic counting are employed in experiments on properties of radioactive substances and their radiations rather than the traditional devices such as electroscopes and electrometers. The latter still occupy an honourable position in the honours degree laboratories of the College.

The subject-matter of this course has its potential hazards; this is emphasized by maintenance of a very high standard of laboratory discipline, with proper emphasis on handling precautions and health safety techniques. Maintenance of this standard is greatly assisted by the clean lines of the light airy rooms.

The laboratory was completed, inexpensively and rapidly, by the conversion of an existing 'Romney hut', the main virtue of which was a sound concrete floor, some $75 \mathrm{ft}$. by $36 \mathrm{ft}$., protected by a semicylindrical water-tight shell of galvanized iron. Insertion of a false ceiling and suitable vertical divisions created rooms of normal shape and voids which provide for certain services and for the

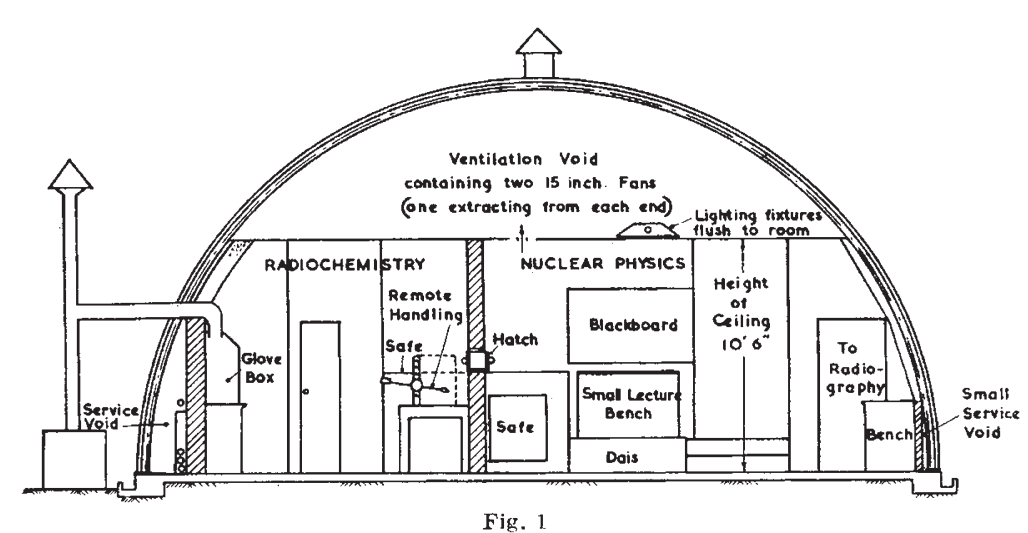

ventilation system, as indicated in the typical trans. verse section of Fig. 1. A substantial central wall divides the building longitudinally into physics and chemistry sections. The physics side contains a nuclear physics laboratory, a gamma-radiography room, a small counting room, a photographic dark room, a small workshop and an office. The subdivision on the chemistry side, based on ascending levels of radioactivity, provides a preparation room, a monitoring room and three radiochemical laboratories in which the anticipated level of activity is graded from negligible to dangerous. This arrangement leads to a gradation of working levels with the potentially quite dangerous activation room on the chemistry side opposite the radiography room, so that both are remote from the shielded counting room and the regions in which prolonged work is performed. The radioactive sources, in suitable individual containers, are kept in massive concrete and iron safes, one in each of the two main divisions.

The rooms are separately ventilated into the roof void above the light-alloy false ceiling, which is stiffened by overhead girders and rests on the partition walls. This system minimizes inter- 
change of active material between the rooms. The flow of air is governed by a pair of powerful fans and it is expelled through the original ventilating shafts on top of the building. The intake is through individual gratings to prevent one room drawing air from another. The chemistry laboratories have an additional air flow when the fume-hoods are in operation. This system could have air-filters added on the outflows if necessary. It should, however, be mentioned that current opinion inclines to make the air-flow downwards because this is assisted by the normal movement of those grosser particles which become airborne.

All exposed wall surfaces are smooth and coated with hard glossy paint, light in colour, so that splashes are easily visible. The concrete floor is completely covered with waxed polished linoleum and the joins sealed. The lighting is concealed, and all services are, whenever possible, out of sight and carried between laboratories above the false roof and inside the vertical walls. In this way surface decontamination is greatly facilitated. The main dividing wall is provided with two lead-lined hatches, so that radioactive sources can be passed from the chemistry laboratory to the physies laboratory without passing through the monitoring room.

All effluent from the monitoring room and the radiochemical laboratories passes into external delay tanks, arranged below ground-level. Such effluent is not finally discharged until the level of activity has been checked as being below the maximum agreed with the relevant authority. It should be noted that public health authorities must be consulted about all arrangements of this kind. Solid waste which might be active is put into paper bags in footoperated medical-type waste bins. These are later sealed, and if passed for disposal, are burnt in an incinerator, remote from any habitation.

There are two neutron sources in the activation room. The larger source, an antimony-beryllium mixture with a nominal gamma-ray activity of 1 curie, is housed in a drum of paraffin wax, surrounded by a concrete cylinder built from interlocking rings with aligned holes which form vertical channels for inserting specimens for irradiation. In this way, tracer quantities of artificially radioactive materials are regularly prepared. The smaller source is of a radium-beryllium mixture, having associated gammaactivity equivalent to $20 \mathrm{mgm}$. of radium. Normally housed in a converted dustbin containing a hollow lead cylinder embedded in paraffin wax, this source is used for physical experiments involving neutrons.

Except in emergency, the monitoring room is the only means of entry to the radiochemical laboratories. Here, all staff and students check themselves for contamination before entering and when leaving the laboratories. The hand-basins have foot-operated taps, and powerful hot-air hand-dryers are used in place of towels, to reduce cross-contamination risks. All personnel wear protective overalls in all the laboratories, but, in addition, rubber gloves and canvas overshoes are worn in the radiochemical laboratories. The intake of radioactive material is so serious a hazard that eating, smoking and the like are strictly forbidden except in the office, which is adjacent to the main entrance. Since familiarity often causes research workers as well as technicians and industrial workers to become lax, and the consequences of mistakes may not show for long periods, it is essential to be rigid in enforcing this discipline, but the reasons for all rules should be fully understood and no avoidable hardship should be inflicted. For example, the hand-dryers must be very efficient so that there is no temptation to hasten operations with a surreptitious use of a handkerchief. Staff and students carry film badges and sometimes also quartz-fibre dosimeters, and a diary is maintained of individual radiation doses so recorded.

The laboratory furniture is normal, but bench tops are protected by stout water-proof waxed paper which can easily be removed after contamination. Chemical experiments are 'contained' in stainless steel trays lined with tissue paper. Normal chemical manipulation is drastically modified, there being no sniffing and tasting, while wash-bottles are replaced by plastic squeeze-bottles and pipettes are pistonoperated. The preparation of sources which are powerful alpha- or beta-emitters is carried out in a glove-box, having rubber gloves sealed into apertures in the 'Perspex' windows: strong gamma-emitters are handled behind an appropriate shielding wall of interlocking lead bricks by remote handling gear. The weight of the walls is taken by the reinforced concrete bench which, incidentally, shields the lower part of the body.

Most of the apparatus on the physics side comprises various forms of radiation detector and counting equipment. The standard counting unit consists of a Geiger-Müller tube housed in a lead 'castle' and connected to a dekatron scaler by a small pre-amplifier and delay circuit. The voltage supply for the Geiger tubes is provided by power packs, each capable of feeding several units. In adidition, three rate-meters are available for the direct measurement of counting rates, using endwindow or liquid detectors. A pen recorder in con. junction with a rate-meter enables the decay curve of some isotopes to be traced directly. Batteryoperated radiation monitors and Radiac equipment are used in simple experiments, apart from serving as laboratory monitors and survey meters. More specialized equipment includes two scintillation counters (one is incorporated in a single-channel gamma-ray spectrometer); two vibrating-reed electrometers, used for alpha-ray range determination and for ionization current measurements, etc. ; and a home-made, thin-lens type, beta-ray spectrometer.

Weak radioactive sources (micro-curies) are prepared by evaporation from nickel planchettes of diluted radioactive solutions obtained from the Radiochemical Centre, Amersham. Infra-red lamps are used for this operation to avoid possible spray. The planchettes are mounted on thin 'Perspex' sheets designed to fit into a lead castle. More active sources (milli-curies) as wire or foil are obtained from Harwell. For gamma-radiography, the main source is of iridium-192 of several curies strength, used in a gamma-ray 'bomb' container. When the stronger sources are used in the exposed state in an experiment, lead brick shielding is used to ensure a reasonably low background throughout the laboratory. It has been found possible to cast suitable lead bricks very cheaply in the college workshops.

The experiments carried out in the physics section include the characteristics of the absorption of alpha-, beta- and gamma-rays; the calibration of dosimeters and of film badges; the survey of contaminated ground; the determination of beta- and gamma-ray spectra; the slow neutron flux obtained from a neutron source immersed in water ; the characteristics 
of Geiger and scintillation counters; the decay curves of irradiated silver; the use of nuclear emulsions; and the production of gamma-radiographs. On the chemistry side, the experiments include the production and isolation of radioactive iodine; slow-neutron activation analyses; isotopic dilution analyses; separation using exchange resins and the solubility of lead sulphate. The experiment schedules are modified to cater for the differing academic levels of students using the laboratory.

The laboratory caters for a maximum of thirty students at a time, of whom about twenty would be on the physical side and the rest doing chemical experiments. This represents a fairly high concentration of personnel in the working space, and constant vigilance is required to prevent excess local back. ground, especially when sources are moved. Writing records of experiments is then usually carried out in a neighbouring building.

The capital cost of conversion, including benching, ventilation and drainage, for a laboratory on this scale, is likely to be in the region of $£ 5,000$; a brandnew building would cost much more and take longer to construct. Equipment at the standard adopted for this laboratory is comparable in cost. It is felt that the high initial cost of carefully chosen commercial apparatus has been fully justified in the sequel by the excellent service it has given and by the low maintenance costs.

We wish to record our indebtedness to those members of the Atomic Energy Research Establishment and the Atomic Weapons Research Establishment, who were consulted during the planning stage, for their valuable advice and to the Garrison Engineer at Shrivenham and his staff for their co-operation and professional skill. To those who contemplate similar ventures we would warmly recommend a close study of laboratories of high standard. There are many points of detail which cannot be included in an account of this kind, but which make a difference to smooth and efficient running of the laboratories and reduce the risk of accident. The close co-operation of physicist and chemist in the design and operation of the laboratory has played a notable part in promoting its efficiency and its usefulness to the students, who gain a more balanced picture of the subject in this way.

\section{THE ELECTRICAL RESEARCH ASSOCIATION}

$\mathrm{T}$ HE Electrical Research Association has recently completed its first year of work, under the direction of Dr. H. G. Taylor, at the new laboratories at Leatherhead. Changes and developments are reflected in some measure in the form as well as in the content of the annual report for $1957^{*}$ which was presented at the annual general meeting held on May 7.

It is with regret that we record the death, on the day of the meeting, of Mr. F. H. Bramwell, chairman of the Council of the Association, over whose signature the report of the Council appears in the annual report.

In the matter of framing research policy it is noteworthy that the Council now has the benefit of drawing upon the knowledge and experience of an Advisory Research Board, the constitution of which is given in the report under the list of committees and panels. On the administrative side a new post of research manager has been established and is occupied by Mr. L. Gosland, who was formerly head of the switch. gear department.

In order to form some assessment of the extent to which the Association's research effort is co-ordinated with the interests of members, a questionnaire was issued during the year inviting opinion as to the application of the Association's research funds. The results were weighted in relation to the value of members' subscriptions and a final percentage analysis prepared. This analysis is found to be in reasonably good correlation with the relative scale of the Association's activity in its various fields, and although it does not show a pressing demand for work in any field in which the Association is not at present engaged, there is a significant emphasis on the subject of the nuclear irradiation of dielectrics, cathodic protection, climate proofing, nuclear power and semi-conductors.

* Electrical Research Association. Thirty-seventh Annual Report for the year ended 31 st December, 1957. Pp. 119+8 plates. (Leather-
The results of this questionnaire provide very useful guidance for future policy.

Mention is made of the difficulties which have been experienced in filling vacancies on the senior scientific staff and of the efforts which are being made to interest young university graduates in engineering and in physics in the work of the Association. The total number of staff employed by the Association at the end of the year under review was 348 .

In the field of insulation, which constitutes one of the major areas of the Association's work, a result of considerable practical significance has been obtained in the demonstration of the importance of moisture content in controlling the discharge inception-level of cellulose. Confirmation has also been obtained, in the case of one of the new high-density polyethylenes, of the recently propounded electro-mechanical theory of breakdown.

It is interesting to note that it has been found desirable to initiate a fundamental study of the mechanism of oxidation of hydrocarbon oils. This programme is being carried out in three university departments. The need to supplement the great body of existing knowledge of the behaviour of hydrocarbon oils as insulants for electrical apparatus arises from the urge to raise the working temperature of transformers.

In another important branch of the Association's work, that of circuit breakers and circuit-breaking phenomena, progress is reported in the breaking of both low and high currents in vacuum and, in relation to the are itself, theoretical predictions of high arccore temperatures $\left(35,000^{\circ} \mathrm{K}\right.$.) have been confirmed by spectroscopic measurements.

Two interesting full-scale experiments relating to electric power systems are reported. These were carried out in collaboration with the Central Electricity Authority. The stability of a large national electricity supply system under conditions of overload or of transient disturbance is naturally a matter of 